\title{
Demonstration of nondeclarative sequence learning in mice: Development of an animal analog of the human serial reaction time task
}

\author{
Michael A. Christie and Steven M. Hersch ${ }^{1}$ \\ MassGeneral Institute for Neurodegenerative Disease, Department of Neurology, Massachusetts General Hospital and Harvard \\ Medical School, Boston, Massachusetts 02129, USA
}

\begin{abstract}
In this paper, we demonstrate nondeclarative sequence learning in mice using an animal analog of the human serial reaction time task (SRT) that uses a within-group comparison of behavior in response to a repeating sequence versus a random sequence. Ten female B6CBA mice performed eleven 96-trial sessions containing 24 repetitions of a 4-trial sequence. During the 12th session, the repeating sequence was replaced with the random sequence halfway through the session. Reaction time (RT) to respond to an illuminated nose-poke was recorded, and performance was compared at the halfway point in each session to test for any change in behavior. For learning effect, RTs decreased over the no-switch repeating-sequence sessions. For interference effect, behavior did not change appreciably at the halfway point during the last repeating-sequence session. However, RTs deteriorated significantly after the switch from repeating to random sequences halfway through session 12 . The mice demonstrated a robust interference effect when switched from repeating to random sequences. This pattern of behavior in humans performing the SRT is interpreted as evidence of nondeclarative sequence learning. The similarity between the human and mouse SRTs will enable more direct comparisons of mouse-human nondeclarative memory behavior and will provide a useful behavioral end-point in mouse-models of basal ganglia dysfunction.
\end{abstract}

In humans, the serial reaction time task (SRT) is a nondeclarative/ implicit memory task that produces sequence learning through repetition of uncued and unannounced serially ordered stimuli. Learning is assessed by observing a deterioration in task performance when a random sequence replaces a regularly repeating sequence (the "interference effect"). Subjects with basal ganglia disorders such as Huntington's disease (Knopman and Nissen 1991; Willingham and Koroshetz 1993), Parkinson's disease (Ferraro et al. 1993; Pascual-Leone et al. 1993; Jackson et al. 1995; Westwater et al. 1998; Sommer et al. 1999; Stefanova et al. 2000) or pharmacologic treatments that affect the basal ganglia (Knopman 1991) demonstrate greatly reduced, or entirely absent, interference effects, indicating compromised implicit learning. These SRT deficits in subjects with basal ganglia dysfunction are not simply due to motor performance dysfunction (Harrington et al. 1990), as subjects with damage restricted to the basal ganglia as a consequence of infarct or hemorrhage are significantly impaired in both a motor and nonmotor version of the SRT (Vakil et al. 2000). Furthermore, Vakil et al. (2000) found that while the basal-ganglia infarct subjects were significantly impaired in the SRT in comparison with intact-control subjects, the two groups did not differ on several tests of declarative (explicit) memory function.

In recent years, there has been an ever growing list of genetic mouse models of basal ganglia function and disorders. These include many knockout mice probing receptor and transporter function (Centonze et al. 2003; Metzger et al. 2002; Viggiano et al. 2003), models relevant to drug abuse (Phillips 2002; Berrendero et al. 2003), and transgenic models of Huntington's disease (Mangiarini et al. 1996; Schilling et al. 1999), Parkinson's disease (Maguire-Zeiss and Federoff 2003; Li et al. 2004), and

'Corresponding author.

E-mail hersch@helix.mgh.harvard.edu; fax (617) 724-1480.

Article published online ahead of print. Article and publication date are at http://www.learnmem.org/cgi/doi/10.1101//m.85404. other basal ganglia disorders (Sriram et al. 2002; Orth and Tabrizi 2003; Volpicelli-Daley et al. 2003). Despite the enormous amount of work that has been devoted to characterizing the neurophysiological and motor phenotypes of these mice, there is limited information about their cognitive phenotypes, and no procedures have been developed to help determine how nondeclarative learning might be compromised by any developing neurodegenerative phenotype. A rodent analog of the SRT was recently developed in rats (Christie and Dalrymple-Alford 2004). Neurologically intact rats demonstrated an ability to learn short (4-trial), medium (8-trial) length sequences, and long (12-trial) sequences. Rats with dorsal caudate lesions displayed a marked impairment in the task in the medium and long sequence length conditions (and a milder impairment in the short sequence condition), whereas rats with dorsal hippocampal lesions were not impaired in the short, medium, or long sequence length conditions. We report the first successful adaptation of the SRT for use in mice. The mouse SRT procedure bears close resemblance to the human SRT task, and provides a test of nondeclarative memory function in mouse models of basal ganglia function and disease.

\section{Results}

\section{Acquisition analysis}

Analysis of the repeating-sequence sessions, and of the first half of session 12 , was conducted by aggregating each session into 10 blocks of 16 trials (five blocks for session 12) and performing a repeated-measures ANOVA. The main effect of block was significant $\left(F_{(9,90)}=13.38, P<0.0001\right)$; the mice responded faster over time (Fig. 1A). However, performance during sequence acquisition is a mixture of changes in general factors such as sensorimotor skills and attention, in addition to any specific sequence learning. In contrast, disruption revealed by the interference effect provides specific evidence of the influence of acquisition of 
A

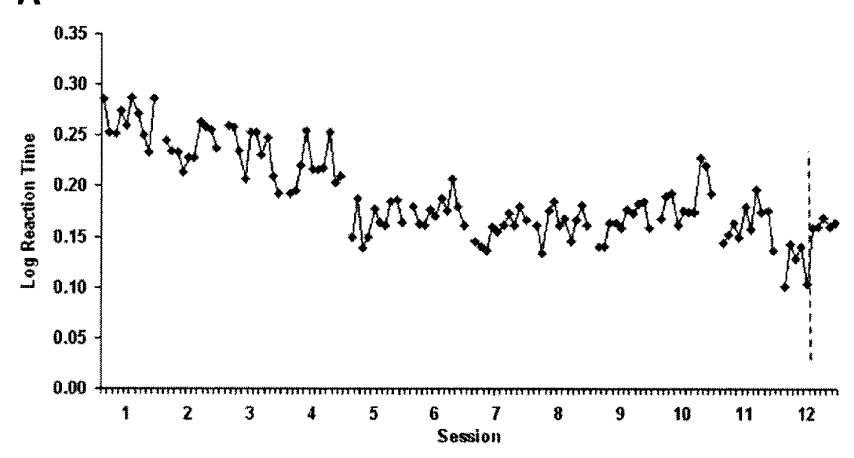

B

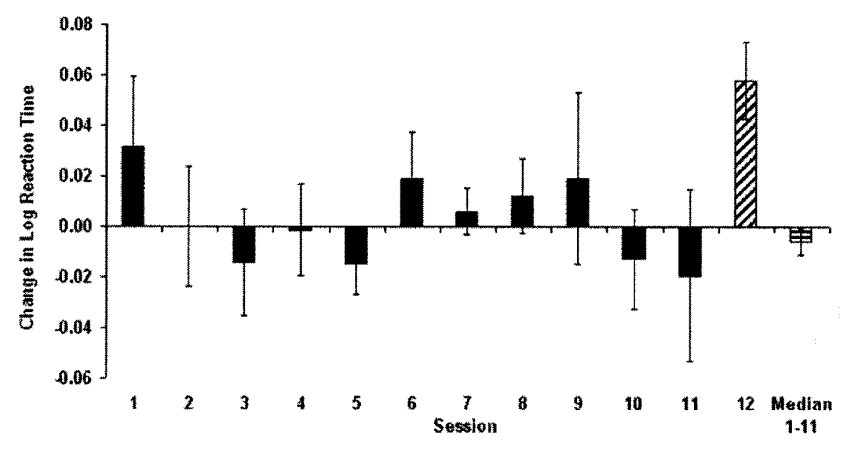

Figure 1. (A) Acquisition performance for log reaction time scores. The first 11 sessions all contained seamless repetitions of the repeating sequence throughout the session. In session 12 the repeating sequence was replaced with random trials at the halfway point (dotted line). (B) Interference effects for log reaction time scores. The first 11 sessions all contained seamless repetitions of the repeating sequence throughout the session. Session 12 contained the repeating sequence for the first half of the session, but thereafter switched to a random sequence for the remainder of the session. Median 1-11 is the median interference effect for the first 11 sessions. Error bars $= \pm 1$ SEM.

the sequence itself, as the only change is the predictability of the sequence of stimuli.

\section{Interference analysis}

The critical comparison is between session 11, the last repeatingsequence session, and session 12 , the switch-to-random session. A one-tailed $t$-test revealed that the interference effect in session 12 was significantly higher than that in session $11(t(17)=2.01$, $P=0.03$; Fig. 1B). Furthermore, single-sample $t$-tests revealed that whereas performance changed significantly at the halfway point in session $12[(t(9)=2.89, P=0.017)$; Fig. $1 \mathrm{~B}]$, the was no change across the halfway point in session $11(P=0.7)$. This pattern of behavior in humans is considered evidence of nondeclarative sequence learning.

The composite interference effect for the 11 repeatingsequence sessions was virtually zero, as would be expected if these effects were simply a result of behavioral variation and were not a significant response to experimental conditions. Furthermore the composite interference effect was significantly smaller than that of the interference effect generated in session 12 $(t(9)=2.53, P=0.02)$.

Error rates were also analyzed for both acquisition and interference effects. However, the mice made so few errors (typically $<5$ per session, during both repeating and random conditions) that there wasn't sufficient data to analyze.

\section{Discussion}

We tested the ability of mice to perform an animal analog of the human SRT task. Interference effects, the 'gold standard' of human SRT studies, were examined across sessions. The mice only displayed an interference effect in the one session within which the repeating sequence was replaced with random sequences. In humans this deterioration in behavior after the switch to random sequences is considered evidence of nondeclarative sequence learning (Nissen and Bullemer 1987; Reber and Squire 1994; Curran 1997).

Unlike other nondeclarative memory/sequence-learning tasks modeled in mice, the mouse SRT closely mirrors the methodology of the human SRT task. In particular, four simple stimulus-response locations used with a specific repeating sequence of stimulus presentations is analogous to the sensorimotor aspects of the human task, and only requires a short, simple motor response. Furthermore, as in human studies, the mice performed mass trials of a repeating sequence before being switched, within session, to a random sequence to provide evidence of sequence learning. Prior work has shown a switch within sessions to be a more sensitive measure of sequence learning than a switch between sessions (M. Christie and J. Dalrymple-Alford, unpubl.). Following Reed and Johnson (1994) we provided equal stimulus probability and made other statistical aspects of frequency information identical between the repeating and random sequences, to ensure that learning the specific repeating sequence alone could result in an interference effect when switched to random sequences.

There is evidence from other food-rewarded protocols that rats can learn serially ordered information. Both caudate and hippocampal lesions disrupt acquisition of serially presented rewards of varying magnitude (Compton 2001); cortical ablation produces a deficit in the performance of serially ordered grooming behavior (Berridge and Wishaw 1992), and striatal lesions in rats impair the acquisition of a repeating sequence of radial-arm maze visits (DeCoteau and Kesner 2000). However, findings from studies which used protocols more directly comparable to the human SRT are unclear. The lack of the critical interference condition in Nixon and Passingham's (2000) 4-trial SRT study with three cerebellar-lesioned monkeys makes it difficult to conclude anything beyond the fact that the monkeys responded more quickly during repeating sequence training. Procyk et al. (2000) report mixed results from a study with two neurologically intact monkeys. One monkey demonstrated an interference effect (RT increase) when switched from a 4-trial repeating sequence to a random sequence, but the other monkey displayed a RT decrease (i.e., performance improved) when switched to the random sequences. Presumed animal nondeclarative memory tasks may also be prone to declarative memory strategies when used with humans (Broadbent et al. 2002). In contrast, the mouse SRT is both highly analogous to the human SRT and produced a very clear indication of sequence learning in the mice in the context of considerable day-to-day variability in performance.

The 4-trial repeating sequence used in this study was identical to that used in the short-sequence condition of an SRT task recently demonstrated in the rat (Christie and Dalrymple-Alford 2004). Both rats and mice display a marked deterioration in performance when switched, uncued, from the 4-trial repeating sequence to a random sequence. An interesting difference between the two studies was that rats made a high number of errors during SRT performance, whereas mice (and humans) make very few errors during task performance. Although error rates were a valuable measure of sequence learning in the rat SRT study, the very low number of errors made by mice rendered the measure unusable in the mouse SRT, which is typically also the case in human SRT performance. It is unclear why rats made so many errors 
during SRT performance and mice didn't, but the difference in motivation in the two studies (intracranial self-stimulation and food reward, respectively) may have played a role.

Studies in patients with basal ganglion disorders have demonstrated a marked impairment in the SRT in comparison with neurologically intact subjects. Parkinson's (PD) and Huntington's diseases (HD), which both disturb basal ganglia function, result in deficits in SRT performance differentiated only by degree. The typical pattern of behavior in PD patients performing the SRT is, firstly, slower reaction times during sequence acquisition, and secondly, and more importantly, a markedly weaker interference effect (compared to controls) when switched from a repeating to a random sequence (Ferraro et al. 1993; PascualLeone et al. 1993; Jackson et al. 1995; Westwater et al. 1998; Sommer et al. 1999; Stefanova et al 2000). Subjects with HD show a similar, but more extreme, impairment in the SRT. Not only do they display minimal improvement in response speed during sequence acquisition, but their interference effect is very weak or entirely absent (Knopman and Nissen 1991; Willingham and Koroshetz 1993). Given the likelihood that cognitive functions begin to deteriorate prior to any motor disturbance in human HD (Lawrence et al. 1998; Paulsen et al. 2001) and that a major aim of therapeutic intervention is to inhibit, if not prevent entirely, the progression of the disease, there is a need for tests of cognitive function in mouse models of HD that are both valid analogs of human nondeclarative memory, and sensitive to early cognitive deterioration resulting from the developing phenotype. The mouse SRT is intended to fill this need, and is particularly intended as an outcome measure for use with mouse models of basal ganglia dysfunction.

Although not as widely accepted or as well detailed as the hippocampus' role in declarative memory, there is general agreement that basal ganglia, and especially the caudate nucleus, are primarily responsible for implicit memory in humans and procedural/reference forms of memory in animals (Packard et al. 1989; Packard and White 1991; Kesner et al. 1993; White 1997; Packard and Teather 1998). However, while these forms of memory are dissociable in rats and the dissociation mimics the hippocampal/basal ganglia distinction found in humans, there is no ready animal analog of nondeclarative memory tasks in humans. Rovee-Collier et al. (2001) argued that tasks in animals typically described as testing nondeclarative memory are better characterized as "not-declarative" by virtue of having no conceptual or operational validity for assaying nondeclarative memory functions beyond not being disturbed by limbic system damage. It is certainly true that tasks typically used to test nondeclarative/ procedural memory in animals (delayed nonmatching to sample, procedural radial-maze protocols, etc.) have few operational points-of-contact with human nondeclarative memory tasks. We now provide a valid mouse analog of a human nondeclarative memory task.

In summary, we report a nondeclarative memory task in mice that is analogous to a task in humans that is sensitive to basal ganglia dysfunction. In addition to modeling nondeclarative memory functions in mice, the mouse SRT provides a useful and relevant method for studying models of basal ganglia function and dysfunction. The mouse SRT has potential as a sensitive outcome measure in genetic mouse models of neurodegenerative diseases, and particularly as a measure of the effect of therapeutic compounds on cognitive dysfunction in such models.

\section{Materials and Methods}

\section{Subjects}

Ten female wild-type mice of the B6CBA background were used. Mice were group-caged and food-restricted to $85 \%$ of their free- food weight. Water was available ad libitum in the home cage, and mice were tested during the light portion of a $12 / \mathrm{h}$ light-dark cycle. The experiment was carried out in accordance with the NIH guidelines for the Care and Use of Laboratory Animals, and the Massachusetts General Hospital IACUC.

\section{Apparatus}

Mice were trained and tested in Med-Associates 9-hole mouse chambers, which were enclosed in individual sound-attenuating cubicles. The chambers were computer controlled by a PC with Med-Associates MED-PC IV behavioral programming software.

\section{Training}

Mice were initially trained to nose-poke four illuminated holes (nos. 2, 4, 6, and 8; left-to-right) in return for a food reward (condensed milk diluted 1:5). After mice achieved baseline criteria (>60 responses in $20 \mathrm{~min}$ ) they were transferred to the SRT task.

\section{SRT Performance}

Mice performed a single 96-trial session per day. The first 11 sessions consisted of 24 repetitions of the 4 -trial repeating sequence (left-to-right; $6,2,8,4$ ). At the halfway point through the twelfth session, the sequence type switched, uncued, from the 4-trial sequence used in the preceding 11 sessions to random sequences. Random sequences were generated as described by Reed and Johnson (1994) to ensure that all frequency information other than the actual repeating sequence was identical between random and repeating sequences. Sets of random sequences were combined as described by Fellows (1967) to create a unique 220 -trial block of random sequences. This 220 -trial block of random trials was used for all mice after all sequenceto-random transitions, and the start position within the block varied randomly for each mouse. Therefore the only difference between sequence and random conditions was the presence or absence of the repeating sequence.

\section{Analysis}

Reaction time (RT) was measured from the time at which the mouse removed its head from the dipper port to the time at which it nose-poked the target (illuminated) hole. All reaction times were log transformed to help adjust for behavioral variance. An interference effect score was calculated for each session at the halfway point during the session, just as if a sequence switch had taken place. This was done by taking the median of the 16 trials immediately prior to, and 16 trials immediately after, the switch point and generating a difference score. A positive value indicated a slowing in RTs. A composite interference effect for all the repeating-sequence sessions (1-11) was also generated and compared to that of session 12 . Interference effects were compared within each of sessions 11 and 12 separately by singlesample $t$-tests to determine whether the effect was different from zero, and between sessions 11 and 12 by dependent means $t$-tests (Statistica).

\section{Acknowledgments}

The authors' research is supported by the NIH (NS35255, AT00613, NS045242, NS045806), the Huntington's Disease Society of America, and The Hereditary Disease Foundation. We thank Dr. Fran Norflus, Dr. Vahnita Chopra, and Mr. Raman Chopra for technical assistance.

\section{References}

Berrendero, F., Castane, A., Ledent, C., Parmentier, M., Maldonado, R., and Valverde, O. 2003. Increase of morphine withdrawal in mice lacking A2a receptors and no changes in CB1/A2a double knockout mice. Eur. J. Neurosci. 17: 315-324.

Berridge, K.C. and Wishaw, I.Q. 1992. Cortex, striatum and cerebellum:

\section{Learning \& Memory}


Control of serial order in a grooming sequence. Exp. Brain Res. 90: $275-290$.

Broadbent, N.J., Clark, R.E., Zola, S., and Squire, L.R. 2002. The medial temporal lobe and memory. In Neuropsychology of memory, 3rd ed. (eds. L.R. Squire and D.L. Schacter), pp. 3-23. Guilford Press, NY.

Centonze, D., Grande, C., Saulle, E., Martin, A.B., Gubellini, P., Pavon, N., Pisani, A., Bernardi, G., Moratalla, R., and Calabresi, P. 2003. Distinct roles of D1 and D5 dopamine receptors in motor activity and striatal synaptic plasticity. J. Neurosci. 23: 8506-8512.

Christie, M.A. and Dalrymple-Alford, J.C. 2004. A new rat model of the human serial reaction time task: Contrasting effects of caudate and hippocampal lesions. J. Neurosci. 24: 1034-1039.

Compton, D.M. 2001. Are memories for stimulus-stimulus associations or stimulus-response associations responsible for serial-pattern learning in rats? Physiol. Behav. 72: 643-652.

Curran, T. 1997. Higher-order associative learning in amnesia: Evidence from the serial reaction time task. J. Cogn. Neurosci. 9: 522-533.

DeCoteau, W.E. and Kesner, R.P. 2000. A double dissociation between the rat hippocampus and medial caudoputamen in processing two forms of knowledge. Behav. Neurosci 114: 1096-1108.

Fellows, B.J. 1967. Chance stimulus sequences for discrimination tasks. Psychol. Bull. 67: 87-92.

Ferraro, F.R., Balota, D.D., and Connor, L.T. 1993. Implicit memory and the formation of new associations in nondementing Parkinson's Disease individuals and individuals with senile dementia of the Alzheimer type: A serial reaction time (SRT) investigation. Brain Cogn. 21: 163-180.

Harrington, D.L., Haaland, K.Y., Yeo, R.A., and Marder, E. 1990. Procedural memory in Parkinson's disease: Impaired motor but not visuoperceptual learning. J. Clin. Exp. Neuropsychol. 12: 323-339.

Jackson, G.M., Jackson, S.R., Harrison, J., Henderson, L., and Kennard, C. 1995. Serial reaction time learning and Parkinson's disease: Evidence for a procedural learning deficit. Neuropsychologia 33: $577-593$.

Kesner, R.P., Bolland, B.L., and Dakis, M. 1993. Memory for spatial locations, motor responses, and objects: Triple dissociation among the hippocampus, caudate nucleus, and extrastriate visual cortex. Exp. Brain Res. 93: 462-470.

Knopman, D. 1991. Unaware learning versus preserved learning in pharmacologic amnesia: Similarities and differences. J. Exp. Psychol. Learn. Mem. Cogn. 17: 1017-1029.

Knopman, D. and Nissen, M.J. 1991. Procedural learning is impaired in Huntington's disease: Evidence from the serial reaction time task. Neuropsychologia 29: 245-254.

Lawrence, A.D., Hodges, J.R., Rosser, A.E., Kershaw, A., French-Constant, C., Rubinsztein, D.C., Robbins, T.W., and Sahakian, B.J. 1998. Evidence for specific cognitive deficits in preclinical Huntington's disease. Brain 121: 1329-1341.

Li, W., Hoffman, P.N., Stirling, W., Price, D.L., and Lee, M.K. 2004. Axonal transport of human $\alpha$-synuclein slows with aging but is not affected by familial Parkinson's disease-linked mutations. J. Neurochem. 88: 401-410.

Maguire-Zeiss, K.A. and Federoff, H.J. 2003. Convergent pathobiologic model of Parkinson's disease. Ann. NY Acad. Sci. 991: 152-166.

Mangiarini, L., Sathasivam, K., Seller, M., Cozens, B., Harper, A., Hetherington, C., Lawton, M., Trottier, Y., Lehrach, H., Davies, S.W., et al. 1996. Exon 1 of the HD gene with an expanded CAG repeat is sufficient to cause a progressive neurological phenotype in transgenic mice. Cell 87: 493-506.

Metzger, R.R., Brown, J.M., Sandoval, V., Rau, K.S., Elwan, M.A., Miller, G.W., Hanson, G.R., and Fleckenstein, A.E. 2002. Inhibitory effect of reserpine on dopamine transporter function. Eur. J. Pharmacol. 456: $39-43$.

Nissen, M.J. and Bullemer, P. 1987. Attentional requirements of learning: Evidence from performance measures. Cogn. Psychol. 19: $1-32$.

Nixon, P.D. and Passingham, R.E. 2000. The cerebellum and cognition: Cerebellar lesions impair sequence learning but not conditional visuomotor learning in monkeys. Neuropsychologia 38: 1054-1072.
Orth, M. and Tabrizi, S.J. 2003. Models of Parkinson's disease. Mov. Disord. 18: 729-737.

Packard, M.G. and Teather, L.A. 1998. Amygdala modulation of multiple memory systems: Hippocampus and caudate-putamen. Neurobiol. Learn. Mem. 69: 163-203.

Packard, M.G. and White, N.M. 1991. Dissociation of hippocampus and caudate nucleus memory systems by posttraining intracerebral injection of dopamine agonists. Behav. Neurosci. 105: 295-306.

Packard, M.G., Hirsch, R., and White, N.M. 1989. Differential effects of fornix and caudate nucleus lesions on two radial maze tasks: Evidence for multiple memory systems. J. Neurosci. 9: 1465-1472.

Pascual-Leone, A., Grafman, J., Clark, B.A., Stewart, M., Massaquoi, S., Lou, J.-S., and Hallett, M. 1993. Procedural learning in Parkinson's disease and cerebellar degeneration. Annals Neurol. 34: 594-602.

Paulsen, J.S., Zhao, H., Stout, J.C., Brinkman, R.R., Guttman, M., Ross, C.A., Como, P., Manning, C., Hayden, M.R., Shoulson, I., et al. 2001. Clinical markers of early disease in persons near onset of Huntington's disease. Neurology 57: 658-662.

Phillips, T. 2002. Animal models for the genetic study of human alcohol phenotypes. Alcohol Res. Health. 26: 202-207.

Proyck, E., Dominey, P.F., Amiez, C., and Joseph, J.P. 2000. The effects of sequence structure and reward schedule on serial reaction time learning in the monkey. Cogn. Brain Res. 9: 239-248.

Reber, P.J. and Squire, L.R. 1994. Parallel brain systems for learning with and without awareness. Learn. Mem. 1: 217-229.

Reed, J. and Johnson, P. 1994. Assessing implicit learning with indirect tests: Determining what is learned about sequence structure. J. Exp. Psychol. Learn. Mem. Cogn. 20: 585-594.

Rovee-Collier, C., Hayne, H., and Colombo, M. 2001. The development of implicit and explicit memory. Advances in consciousness research. John Benjamins Publishing Company, Amsterdam, Philadelphia.

Schilling, G., Becher, M.W., Sharp, A.H., Jinnah, H.A., Duan, K., Kotzuk, J.A., Slunt, H.H., Ratovitski, T., Cooper, J.K., Jenkins, N.A., et al. 1999. Intranuclear inclusions and neuritic aggregates in transgenic mice expressing a mutant N-terminal fragment of huntingtin. Hum. Mol. Genet. 8: 397-407.

Sriram, K., Matheson, J.M., Benkovic, S.A., Miller, D.B., Luster, M.I., and $\mathrm{O}^{\prime}$ Callaghan, J.P. 2002. Mice deficient in TNF receptors are protected against dopaminergic neurotoxicity: Implications for Parkinson's disease. FASEB J. 16: 1474-1476. Epub 2002 July 18.

Sommer, M., Grafman, J., Clark, K., and Hallett, M. 1999. Learning in Parkinson's disease: Eyeblink conditioning, declarative learning and procedural learning. J. Neurol. Neurosurg. Psychiatry 67: 27-34.

Stefanova, E.D., Kostic, V.S., Ziropadja, L., Markovic, M., and Ocic, G.G. 2000. Visuomotor skill learning on serial reaction time task in patients with early Parkinson's disease. Mov. Disord. 15: 1095-1103.

Vakil, E., Kahan, S., Huberman, M., and Osimani, A. 2000. Motor and non-motor sequence learning in patients with basal ganglia lesions: The case of serial reaction time (SRT). Neuropsychologia 38: 1-10.

Viggiano, D., Ruocco, L.A., and Sadile, A.G. 2003. Dopamine phenotype and behaviour in animal models: In relation to attention deficit hyperactivity disorder. Neurosci. Biobehav. Rev. 27: 623-637.

Volpicelli-Daley, L.A., Hrabovska, A., Duysen, E.G., Ferguson, S.M., Blakely, R.D., Lockridge, O., and Levey, A.I. 2003. Altered striatal function and muscarinic cholinergic receptors in acetylcholinesterase knockout mice. Mol. Pharmacol. 64: 1309-1316.

Westwater, H., McDowall, J., Siegert, R., Mossman, S., and Abernathy, D. 1998. Implicit learning in Parkinson's disease: Evidence from a verbal version of the serial reaction time task. J. Clin. Exp. Neuropsychol. 20: 413-418.

White, N.M. 1997. Mnemonic functions of the basal ganglia. Curr. Opin. Neurobiol. 7: 164-169.

Willingham, D.B. and Koroshetz, W.J. 1993. Evidence for dissociable motor skills in Huntington's disease patients. Psychobiology 21: $173-182$.

Received May 7, 2004; accepted in revised form October 5, 2004. 


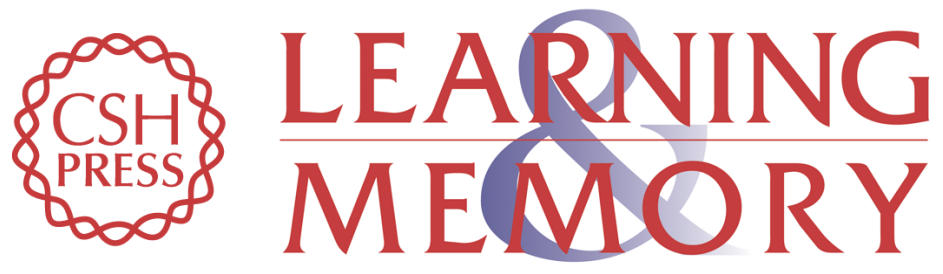

\section{Demonstration of nondeclarative sequence learning in mice: Development of an animal analog of the human serial reaction time task}

Michael A. Christie and Steven M. Hersch

Learn. Mem. 2004, 11:

Access the most recent version at doi:10.1101//m.85404

References This article cites 40 articles, 7 of which can be accessed free at: http://learnmem.cshlp.org/content/11/6/720.full.html\#ref-list-1

License

Email Alerting

Receive free email alerts when new articles cite this article - sign up in the box at the Service top right corner of the article or click here. 